\title{
Is the human masticatory system devoid of recurrent inhibition?
}

\author{
K. S. Türker • A. Schmied • A. Rossi • \\ R. Mazzocchio • P. F. Sowman • J. P. Vedel
}

Published online: 11 January 2007

(C) Springer-Verlag 2007

\section{Erratum to: Exp Brain Res}

\section{DOI 10.1007/s00221-006-0774-2}

In Table 1, header lower part, the word "L-Acetylcarnitine" should read "Saline". The correct Table is given here:

The online version of the original article can be found at http://dx.doi.org/10.1007/s00221-006-0774-2.

K. S. Türker $(\bowtie) \cdot$ P. F. Sowman

Research Centre for Human Movement Control,

Discipline of Physiology, School of Molecular

and Biomedical Sciences, University of Adelaide,

Adelaide, SA 5005, Australia

e-mail: kemal.turker@adelaide.edu.au

A. Schmied $\cdot$ J. P. Vedel

P3M-CNRS, 31 Chemin Joseph Aiguier,

13402 Marseille Cedex 20, France

A. Rossi · R. Mazzocchio

Unità di Neuroficsiologia Clinicia,

Università degli Studi di Siena,

Dipartimento di Scienze Neurologiche e del

Comportamento, Policinico Universitario Le Scotte,

viale Bracci, 53100 Siena, Italy 
Table 1 Comparison of measured variables

\begin{tabular}{|c|c|c|c|c|}
\hline L-Acetylcarnitine & Pre-injection & Injection & Post-injection & Manova (P) \\
\hline Motor unit pairs $(N)$ & 16 & 16 & 15 & \\
\hline Significant synchronization $(N)$ & 16 & 13 & 14 & \\
\hline Peak width (ms) & $9.7 \pm 6.7$ & $11.1 \pm 9.9$ & $11.3 \pm 7.1$ & 0.46 \\
\hline Peak area (imp./trigger) & $0.033 \pm 0.016$ & $0.025 \pm 0.015$ & $0.035 \pm 0.013$ & 0.13 \\
\hline CIS (imp./s) & $0.36 \pm 0.15$ & $0.29 \pm 0.18$ & $0.40 \pm 0.17$ & 0.2 \\
\hline$K^{\prime}$ & $1.33 \pm 0.17$ & $1.28 \pm 0.17$ & $1.27 \pm 0.12$ & 0.36 \\
\hline Background EMG (EMG max \%) & $7.5 \pm 4.5$ & $7.5 \pm 4.1$ & $7.5 \pm 3.8$ & 0.96 \\
\hline ISI (geometric mean, ms) & $73.9 \pm 12.5$ & $68.9 \pm 11.4$ & $68.7 \pm 11.4$ & NS \\
\hline ISI CV (geometric mean, \%) & $27 \pm 5$ & $26 \pm 6$ & $27 \pm 8$ & NS \\
\hline Saline & Pre-injection & Injection & Post-injection & Manova (P) \\
\hline Motor unit pairs $(N)$ & 16 & 16 & 15 & \\
\hline Significant synchronization $(N)$ & 12 & 14 & 11 & \\
\hline Peak width (ms) & $10.6 \pm 5.5$ & $10.8 \pm 5.4$ & $9.8 \pm 3.6$ & 0.98 \\
\hline Peak area (imp./trigger) & $0.030 \pm 0.016$ & $0.028 \pm 0.016$ & $0.026 \pm 0.015$ & 0.73 \\
\hline CIS (imp./s) & $0.35 \pm 0.22$ & $0.32 \pm 0.19$ & $0.33 \pm 0.25$ & 0.72 \\
\hline$K^{\prime}$ & $1.33 \pm 0.19$ & $1.27 \pm 0.18$ & $1.24 \pm 0.15$ & 0.27 \\
\hline Background EMG (EMG max \%) & $6.7 \pm 5$ & $6.7 \pm 4.7$ & $7.3 \pm 6.3$ & 0.49 \\
\hline ISI (geometric mean, ms) & $76.7 \pm 15$ & $74.4 \pm 15.3$ & $72.9 \pm 11.9$ & NS \\
\hline ISI CV (geometric mean, \%) & $28 \pm 6$ & $28 \pm 8$ & $30 \pm 7$ & NS \\
\hline
\end{tabular}

